\title{
Isolated Tibial Component Failure in Total Knee Arthroplasty: A Case Series Evaluating Inflammatory Response versus Mechanical Failure
}

\author{
Sanar S. Yokhana, MD ${ }^{1,2}$ Christopher Bergum, BS² \\ ${ }^{1}$ Department of Orthopaedic Surgery, Detroit Medical Center, \\ Detroit, Michigan \\ 2 Department of Orthopaedic Surgery, Providence-Providence Park \\ Hospital, Southfield, Michigan \\ ${ }^{3}$ Department of Biomedical Engineering, Wayne State University, \\ Detroit, Michigan \\ ${ }^{4}$ Department of Orthopaedic Surgery, St. John-Providence Hospital, \\ Southfield, Michigan \\ J Knee Surg 2019;32:659-666.
}

Weiping Ren, MD, PhD 3 David C. Markel, MD

\begin{abstract}
Keywords

- total knee arthroplasty

- Scorpio knee

- revision knee arthroplasty

- implant failure

Total knee prostheses are routinely redesigned to improve performance, longevity, and to closer mimic the native kinematics of the knee. Despite continued improvements, all knee implants, even those with proven design features, have failures. We identified a cohort of patients with isolated tibial component failures that occurred in a popular and successful knee system. Our purpose was to (1) characterize the observed radiographic failure pattern; (2) investigate the biologic response that may have contributed to the failure; and (3) to determine if the failure mechanism was of a biological or a mechanical nature. Twenty-one knees from 19 patients met the inclusion criteria of isolated tibial component failure in a single knee implant system. Radiographs from the primary and revision knee surgery were analyzed for implant positioning and the failure pattern. Inflammatory biomarkers interleukin (IL)-1 $\beta$, IL-6, and tumor necrosis factor (TNF)- $\alpha$ were available in $16 / 21$ knees and peripheral $C D 14^{+} / 16^{+}$monocytes were measured in 14 of the aforementioned 16 knee revisions. Serum CD3, CD4, and CD19 were measured in 10 of the aforementioned 14 knees. Additionally, white blood cell (WBC) count, erythrocyte sedimentation rate (ESR), and C-reactive protein (CRP) were measured to rule out infection as a cause of the cytokine upregulation. Radiographic findings demonstrated that all of the 21 tibial components were implanted in either neutral or 2 to 3 degrees varus in the coronal plane, and none of the revisions was implanted in valgus. All tibias showed obvious radiographic loosening and the implant failed into varus. The inflammatory biomarkers IL-1 $\beta$, IL-6, and TNF- $\alpha$ were negative. WBC, ESR, and CRP were normal. Serum CD3, CD4, and CD19 flow cytometry analyses were found to be in the normal range. Peripheral $\mathrm{CD} 14^{+} / 16^{+}$and total $\mathrm{CD} 16^{+}$ monocytes measurements were consistent with previous findings of patients with osteoarthritis, rather than particulate-induced inflammatory loosening. The findings support the implant failure observed in our study occurred by a different mechanism than the wear debris-induced aseptic loosening. We believe that a mechanical failure
\end{abstract}

\author{
Address for correspondence David C. Markel, MD, Department of \\ Orthopaedic Surgery, Providence-Providence Park Hospital, 16001 \\ West Nine Mile Road, Southfield, MI 48075-4818 \\ (e-mail: david.markel@ascension.org).
}

received

June 8,2017

accepted after revision

May 26, 2018

published online

July 10, 2018
Copyright @ 2019 by Thieme Medical Publishers, Inc., 333 Seventh Avenue, New York, NY 10001, USA. Tel: +1(212) 584-4662.
DOI https://doi.org/

10.1055/s-0038-1666831. ISSN 1538-8506. 
can occur based on our findings. The loosening, collapse, and debonding from the cement may have been related to the implantation technique, stresses due to favorable rotational freedoms of the implant, or patient characteristics/behavior. Continued exploration into implant failure mechanisms and particularly into the biologic response associated with failure is ongoing.

Total knee arthroplasty (TKA) is a popular and effective treatment for those who suffer from end-stage arthritis. ${ }^{1-4}$ Based on population samples and growth in the United States, it is projected that the number of TKAs will increase $673 \%$ and revision TKA to increase $601 \%$ by the year $2030 .{ }^{5}$ Knee implant designs continue to evolve with the aim of better reproducing the kinematics of the natural knee, to allow increased flexion, and to improve implant fixation and longevity. The desire to improve kinematics led to the creation of a single-radius femoral component design that was introduced by Stryker Orthopaedics in 1996. The Stryker Scorpio Single Axis Knee System offered a constant center of rotation throughout the range of motion with the goal of providing uniform ligament tension during the transition from extension to flexion. The Scorpio Knee was quite successful and had clinically satisfactory outcomes compared with other knee systems on the market. $^{6-14}$ Despite its popularity and generally excellent outcomes, there were a few reports of early tibial failures due to aseptic loosening (AL). ${ }^{11,13} \mathrm{AL}$ has been described as a slowly advancing process in which biomaterial wear debris creates an inflammatory response, albeit low grade. ${ }^{15}$ Polymethylmethacrylate (PMMA) and polyethylene particulates elicit macrophage-mediated inflammatory responses that are characterized by increased release of tumor necrosis factor (TNF)- $\alpha$, interleukin (IL)-1 $\beta$, and IL-6. ${ }^{16-19}$ Additionally, the inflammatory mediators, peripheral $\mathrm{CD}_{1} 4^{+} / \mathrm{CD} 16^{+}$monocytes, CD3, CD4, and CD19 lymphocytes produce a panel of proinflammatory cytokines. These cytokines are of central importance in the pathogenesis of inflammatory diseases including implant loosening. ${ }^{20-25}$ Interestingly, the pattern of failure and tissue response of the failed Scorpio tibial components described herein did not appear to demonstrate the usual characteristics of AL resulting from wear debrisinduced inflammation. Therefore, we set out to investigate the biologic response in a unique cohort of 19 patients (21 knees) who exhibited isolated failure of the tibial component. Intraoperatively, the failure pattern was unusual because the tibial component appeared to be a "debonded" from the cement rather than having the more typical wear debris-associated pattern of AL and/or failure at the bone-cement interface. Our purpose was to (1) characterize the observed radiographic failure pattern; (2) investigate the biologic response that may have contributed to the failure; and (3) determine if the failure mechanism was of a biological or a mechanical nature.

\section{Methods}

Study approval was obtained through the hospital's Institutional Review Board; 19 patients (21 knee revisions) met the criteria of having a revision of a Stryker Scorpio PS Knee arthroplasty as a result of isolated tibial component failure. All primary and revision knee arthroplasty procedures were performed by the senior investigator. All primary and revision implants were cemented using the Stryker Simplex P cement. Cement was mixed in a vacuum mixer per manufactures recommendations. The bone was cleaned with pulse lavage and dried. The proximal tibia was coated with cement and the cement was pressed evenly into the bone. ${ }^{26}$ The cement was also coated onto the entire keel of the tibial component. The implants were impacted into the tibia and compression was maintained across the joint during curing phase of cement with leg in extension.

At the time of revision, the retrieved tibial components underwent gross visual inspection for patterns of wear and/ or fracture. The primary TKAs occurred between 1999 and 2009 and revision surgery occurred between 2008 and 2015. Medical and radiographic patient data were retrieved from the hospital and office charts. Patient demographics such as age, gender, body mass index (BMI), date of primary surgery, and date of revision surgery were obtained.

\section{Radiographic Analysis}

Standing knee radiographs were retrieved from the initial postoperative office visit following the primary TKA to determine if the tibial implants were placed in a neutral, varus, or valgus position. The alignment of the tibial component was evaluated by anteroposterior and lateral radiographic views. The angle of the tibial component was measured from the tibial base to the mechanical axis of the tibia using a goniometer either electronically through the radiographic viewing program or by hand with physical film. Additionally, patient radiographs taken just prior to the revision TKA were reviewed for signs of osteolysis and patterns of implant loosening. All radiographs were reviewed and the findings were recorded by the primary and senior authors.

\section{Biologic Response Analysis}

Peripheral blood was collected prior to revision surgery for white blood cell (WBC) count, erythrocyte sedimentation rate (ESR), and C-reactive protein (CRP) levels. Whole blood and serum samples obtained at the time of surgery were analyzed by flow cytometry and enzyme-linked immunosorbent assay (ELISA). Additionally, joint fluid and tissue cultures were collected intraoperatively at the time of revision surgery to rule out infection as a cause of failure.

Flow Cytometry: Monocytes were characterized based on staining for CD14 and CD16 markers. B cell lymphocyte numbers were determined by CD19 expression and T cell 
lymphocyte numbers were determined by $\mathrm{CD} 3$ and $\mathrm{CD} 4$ expression. Staining of whole blood samples was done per lysing reagent manufacturer's procedure. Briefly, $100 \mu \mathrm{L}$ of whole blood was incubated with various antibodies for 20 minutes. Red blood cells were lysed, and then neutralized with reagents from the ImmunoPrep Reagent System (7546946, Beckman Coulter, Brea, CA) while vortexed for 12 seconds each. Antibodies used were fluorescein isothiocyanate (FITC) mouse antihuman CD14 (peripheral blood monocytes), (clone M5E2, 555397, BD Pharmingen, San Diego, CA), phycoerythrin (PE) mouse antihuman CD16 (peripheral blood monocyte) (clone 3G8, 555407, BD Pharmingen), PE-Cy mouse antihuman CD4 (T-helper cells) (clone SK3, 348789, BD Biosciences), FITC mouse anti-human CD3 (T cell) (clone UCHT1, 11-0038, eBioscience San Diego, CA), and PE mouse antihuman CD19 (B cell) (Clone HIB19, 12-0199, eBioscience). Blood samples were collected on a BD Accuri C6 flow cytometer. Data were analyzed using the BD Accuri C6 software. Based on forward versus side scatter plots, gates were created to include only monocytes or lymphocytes. Unstained controls and samples lacking only the antibody of interest were used to create markers distinguishing negative- and positive-stained samples.

ELISA: TNF- $\alpha$, IL-1 $\beta$, and IL- 6 were evaluated with ELISA. The following ELISA kits were used: TNF- $\alpha$ (DTA00C, R\&D
Systems, Minneapolis, MN), IL-1ß (557966, BD Bioscience, San Diego, CA), and IL-6 (DY206-05, R\&D Systems). ELISA assays were performed according to manufacturer's instructions and plates were read using a BioTek Synergy HT Microplate photometer along with BioTek KC4 software.

\section{Results}

Fifteen female and 4 male patients met the inclusion criteria ( - Table 1 ). The mean age at the time of revision surgery was $68 \pm 9.0$ years (range: 55-86 years), and mean BMI was $35 \pm 6.3$ (range: $25-45$ ). The mean number of years before revision surgery was $6.8 \pm 3.2$ years (range: $2.3-14.4$ years). All patient underwent primary TKA using the standard medial parapatellar approach and were implanted using PMMA.

\section{Radiographic Assessment}

Radiographic imaging immediately following the primary TKA was available in 17/21 knees (the missing four films had been purged from the system). Of these 17 cases, 12 were implanted in slight varus (2-3 degrees) and 5 knees were implanted in a neutral position. Prior to revision surgery, anterior-posterior and lateral radiographs were reviewed for all 21 knees. All of the patients showed collapse of the tibial component into varus, with collapse of the medial cortex and

Table 1 Patient demographics and radiographic assessment

\begin{tabular}{|l|l|l|l|l|l|}
\hline $\begin{array}{l}\text { Case } \\
\text { number }\end{array}$ & $\begin{array}{l}\text { Age at revision } \\
\text { surgery (y) }\end{array}$ & Gender & $\begin{array}{l}\text { Body mass } \\
\text { index }\end{array}$ & $\begin{array}{l}\text { Time in } \\
\text { situ (mo) }\end{array}$ & $\begin{array}{l}\text { Implanted angle } \\
\text { (deg of varus/valgus) }\end{array}$ \\
\hline 1 & 69 & M & 32 & 40 & 3 deg varus \\
\hline $2^{\text {a }}$ & 69 & F & 46 & 111 & 3 deg varus \\
\hline $3^{\text {a }}$ & 69 & F & 44 & 107 & 3 deg varus \\
\hline 4 & 56 & F & 40 & 61 & Neutral \\
\hline 5 & 78 & F & 30 & 28 & Neutral \\
\hline 6 & 62 & M & 29 & 127 & N/A \\
\hline 7 & 80 & F & 30 & 70 & N/A \\
\hline 8 & 75 & F & 35 & 57 & Neutral \\
\hline 9 & 61 & F & 27 & N/A & N/A \\
\hline 10 & 83 & F & 32 & 77 & 3 deg varus \\
\hline 11 & 87 & F & 35 & 163 & N/A \\
\hline 12 & 55 & F & 27 & 93 & 3 deg varus \\
\hline 13 & 69 & F & 25 & 68 & 2 deg varus \\
\hline 14 & 60 & F & 45 & 59 & 3 deg varus \\
\hline 15 & 62 & M & 31 & 74 & 3 deg varus \\
\hline 16 & 67 & M & 43 & 84 & 3 deg varus \\
\hline 17 & 58 & F & 39 & 66 & Neutral \\
\hline 18 & 66 & F & 41 & 173 & Neutral \\
\hline $19^{\text {a }}$ & 62 & F & 36 & 49 & 2 deg varus \\
\hline $20^{\text {a }}$ & 62 & F & 34 & 48 & 3 deg varus \\
\hline 21 & 75 & F & 31 & 85 & \\
\hline & & & & & \\
\hline
\end{tabular}

${ }^{a}$ Same patient w/bilateral tibial component revision.

${ }^{\mathrm{b}}$ Previous tibial fracture. 
tibial component lift-off laterally. Additionally, lateral radiographs demonstrated tilting in the anterior-posterior direction in all knees along with a halo-like loosening pattern around the keel of the tibial implant indicating implant motion (-Figs. 1 and 2).

\section{ELISA}

Serum samples were negative for TNF- $\alpha$ and IL-1 $\beta$ in all cases. IL-6 analysis demonstrated a wide range of values with a median $19.1 \mathrm{pg} / \mathrm{mL}$. - Table 2 demonstrates preoperative levels of serum WBC count, ESR, and CRP, IL-6, joint fluid WBC count, and polymorphonuclear cells at the time of revision surgery. There were seven cases that had elevated levels of IL-6 but all other markers were negative except for case 7 in which ESR and CRP were mildly elevated as well as IL-6 levels; however, intraoperative assessment indicated no signs of infection and cultures were negative for all patients.

\section{Flow Cytometry}

To assess if there were differences in inflammatory monocyte populations compared with historical $\mathrm{AL}$ or mechanical failure patients, peripheral blood leukocytes of 14 patients were stained with CD14 and CD16 antibodies to analyze the percentage of inflammatory monocytes (10 patients were available for a preliminary experiment to analyze the percentage of $\mathrm{T}$ cells, B cells, and memory vs. naïve $\mathrm{CD} 4^{+} \mathrm{T}$ cells). As shown in -Fig. 3, the mean percentage of inflammatory $\mathrm{CD} 14^{+} \mathrm{CD} 16^{+}$monocytes was $6.37 \pm 2.72 \%(\mathrm{SD})$. When combined with $\mathrm{CD} 14^{+} \mathrm{CD} 16^{+}$population, the mean percentage of the total $\mathrm{CD}_{1} 6^{+}$inflammatory monocyte population was $18.08 \pm 6.79 \%$ (SD). No association was observed between any of the inflammatory monocytes measured among ESR, CRP, and IL-6 levels (data not shown). Analysis of $\mathrm{CD}^{+} \mathrm{T}$ cell and $\mathrm{CD}_{19}{ }^{+} \mathrm{B}$ cell lymphocyte percentages and numbers ( - Fig. 4) was within normal ranges. ${ }^{27}$ A range of percentages
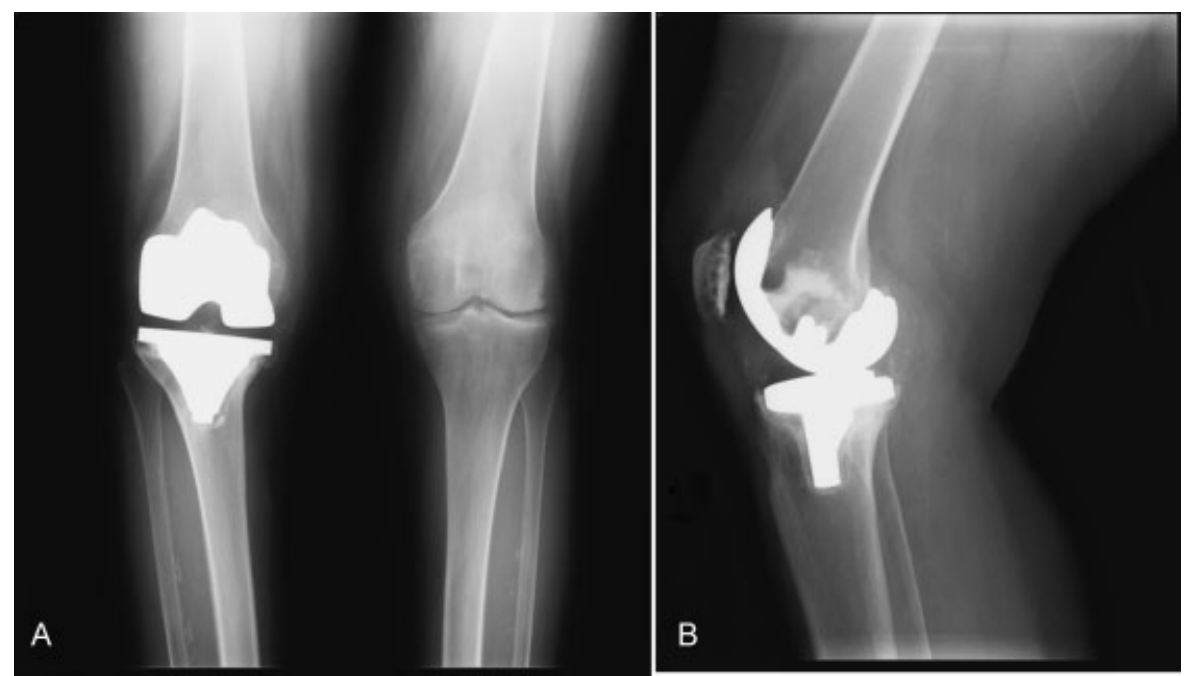

Fig. 1 (A) Anterioposterior image demonstrating medial cortex collapse, lateral lift-off of the tibial tray and loosening around implant. All tibial component failures occurred with the tibial in varus. (B) Lateral image demonstrating radiolucency around the tibial stem.

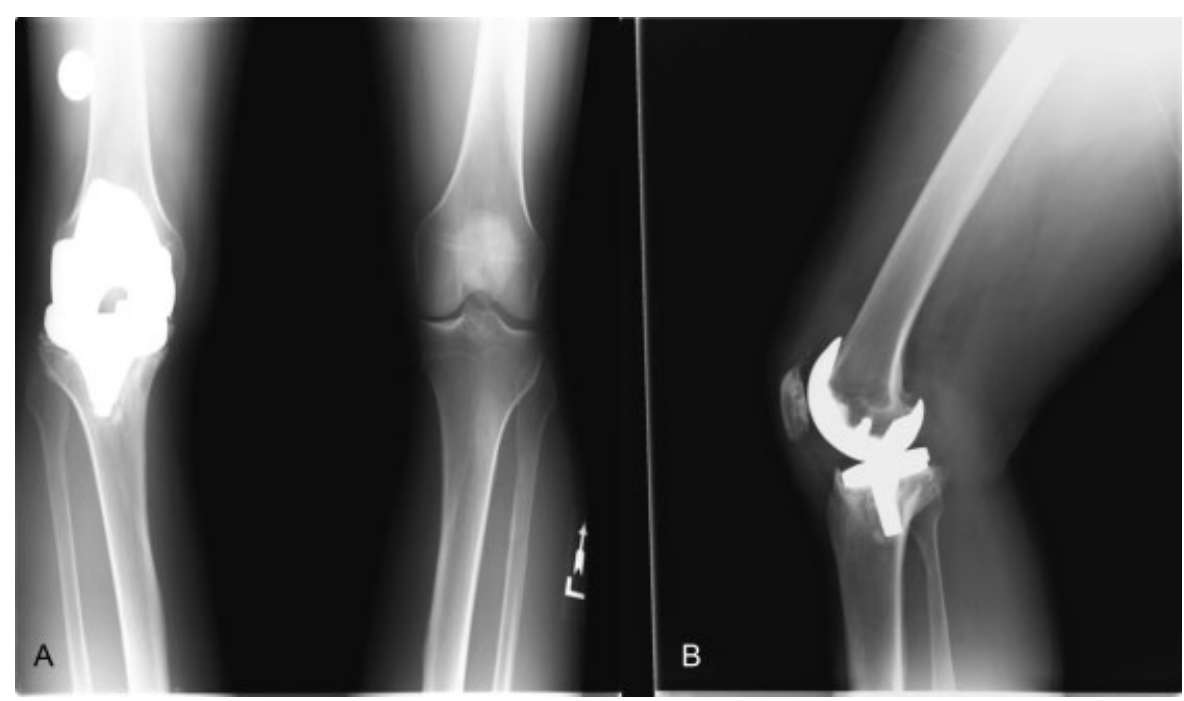

Fig. 2 (A) Anterioposterior image demonstrating medial cortex collapse, lateral lift-off of the tibial tray and loosening around implant. All tibial component failures occurred with the tibia in varus. (B) Lateral image demonstrating radiolucency around the tibial stem. 
Table 2 Serum and joint fluid analysis

\begin{tabular}{|l|l|l|l|l|l|l|l|}
\hline $\begin{array}{l}\text { Case } \\
\text { number }\end{array}$ & $\begin{array}{l}\text { Serum } \\
\text { WBC }\end{array}$ & $\begin{array}{l}\text { Serum } \\
\text { CRP }\end{array}$ & $\begin{array}{l}\text { Serum } \\
\text { ESR }\end{array}$ & $\begin{array}{l}\text { Serum } \\
\text { IL-6 (pg/mL) }\end{array}$ & $\begin{array}{l}\text { Joint fluid } \\
\text { WBC }\end{array}$ & $\begin{array}{l}\text { Joint fluid } \\
\text { \% PMNs }\end{array}$ & $\begin{array}{l}\text { Joint fluid and } \\
\text { tissue cultures }\end{array}$ \\
\hline 1 & 8.8 & 0.3 & 28 & - & 260 & 44 & Negative \\
\hline 2 & 9.3 & 0.2 & 33 & - & 473 & 19 & Negative \\
\hline 3 & 7.4 & 0.2 & $33(\mathrm{H})$ & 6.65 & 1,080 & 17 & Negative \\
\hline 4 & 5.9 & $<0.1$ & 17 & 4.85 & 350 & 5 & Negative \\
\hline 5 & 7.2 & 0.2 & 22 & 165 & - & - & Negative \\
\hline 6 & 5.8 & $<0.3$ & 0 & 696.4 & 381 & 2 & Negative \\
\hline 7 & 6.3 & $1.9(\mathrm{H})$ & $35(\mathrm{H})$ & 1183 & 311 & 48 & Negative \\
\hline 8 & 11.6 & 0.2 & 6 & 0.16 & 1,071 & 50 & Negative \\
\hline 9 & 6.8 & - & - & 19.26 & 217 & 14 & Negative \\
\hline 10 & 13 & 0.2 & $33(\mathrm{H})$ & 83.77 & 800 & 2 & Negative \\
\hline 11 & 6.6 & 0.4 & 11 & 0 & 527 & 8 & Negative \\
\hline 12 & 4.9 & 2.1 & 7 & 75.84 & 322 & 2 & Negative \\
\hline 13 & 5.8 & 0.1 & 6 & 0.88 & 533 & 11 & Negative \\
\hline 14 & 6.8 & - & - & 1.96 & 971 & 69 & Negative \\
\hline 15 & 8.4 & 0.8 & 11 & 19.08 & - & - & Negative \\
\hline 16 & 5.2 & $<0.3$ & 3 & 2.68 & 417 & 8 & Negative \\
\hline 17 & 6.2 & $1.6(\mathrm{H})$ & $28(\mathrm{H})$ & 45.21 & 161 & 11 & Negative \\
\hline 18 & 9 & - & - & 8.45 & 175 & 10 & Negative \\
\hline 19 & 7.1 & $1.6(\mathrm{H})$ & 1 & 21.6 & - & - & Negative \\
\hline 20 & 6.5 & $1.6(\mathrm{H})$ & 1 & 23.05 & 470 & 20 & Negative \\
\hline 21 & 7.49 & .43 & 14 & - & 681 & 28 & Negative \\
\hline
\end{tabular}

Abbreviations: CRP, C-reactive protein; ESR, erythrocyte sedimentation rate; IL, interleukin; PMNs, polymorphonuclear neutrophils; WBC, white blood cell; -, data could not be retrieved.

were observed for $\mathrm{CD}^{+} \mathrm{T}$ cell naïve, effector memory, and central memory cells ( - Fig. 4). ${ }^{27}$ There was no association between naïve and/or memory cell percentages to inflammatory monocytes or other inflammatory markers evaluated.

\section{Discussion}

The Scorpio knee system has had great clinical success and may serve as an example of an excellent knee design. Unfortunately, all implants designs have some failures. In the small cohort of patients studied herein, the lack of inflammatory markers implied that the mechanism of loosening was different than what is typically described in particle-induced AL. This cytokine and tissue response was relatively benign and would not indicate an inflammatory (AL) pattern of loosening. Previous publications have observed isolated failures of the Scorpio tibial component. $6,7,10,11,13$ Based on their findings, it appeared that the majority of failures occurred at the cement-bone interface. Martin et al speculated that the large Deltafit Keel, a unique design feature of the Scorpio system, may be a possible cause for the anecdotal reports of tibial component failure. ${ }^{13}$ Despite this observation, their follow-up showed an overall survivorship rate of $96.4 \%$ at 14 years with only 7 out of 196 patients required revision. Mahoney and Kinsey in their 5- to 9-year follow-up study described an overall survivorship rate of $95.8 \%{ }^{11}$ Both Martin et al and Mahoney and Kinsey observed that all of the failures resulted in a marked varus deformity and that the failure occurred between the cement mantle and bone interface. Additionally, Martin et al speculated that the failures observed may have resulted from implant malpositioning. Interestingly, a majority of the failures observed in our study were placed in slight tibial varus at the time of primary surgery (2-3 degrees) which may have contributed to the mechanical failure. None of the failures were implanted in valgus. In this cohort, all patients were symptomatic with pain (particularly on the medial side) and/or instability. Radiographically, the failure pattern showed tibial component collapse into varus and what appeared to be an anterior-posterior "rocking" represented by a halo around the keel on the lateral radiograph (-Fig. 3). Intraoperatively, the tibial component was devoid of most cement when extracted. This was similar to studies published by Arsoy et al and Foran et al that described tibial debonding in the Zimmer Nexgen LPS and MIS systems, respectively. ${ }^{28,29}$ Arsoy et al, in their study based on 25 tibial component failures, described a similar failure pattern (tibial failure into varus) and found no correlation between the implant's positioning at the time of the primary TKA. Foran et al speculated that the etiology of the tibial component debonding may be attributed to one or more of the following factors: surgical technique, 

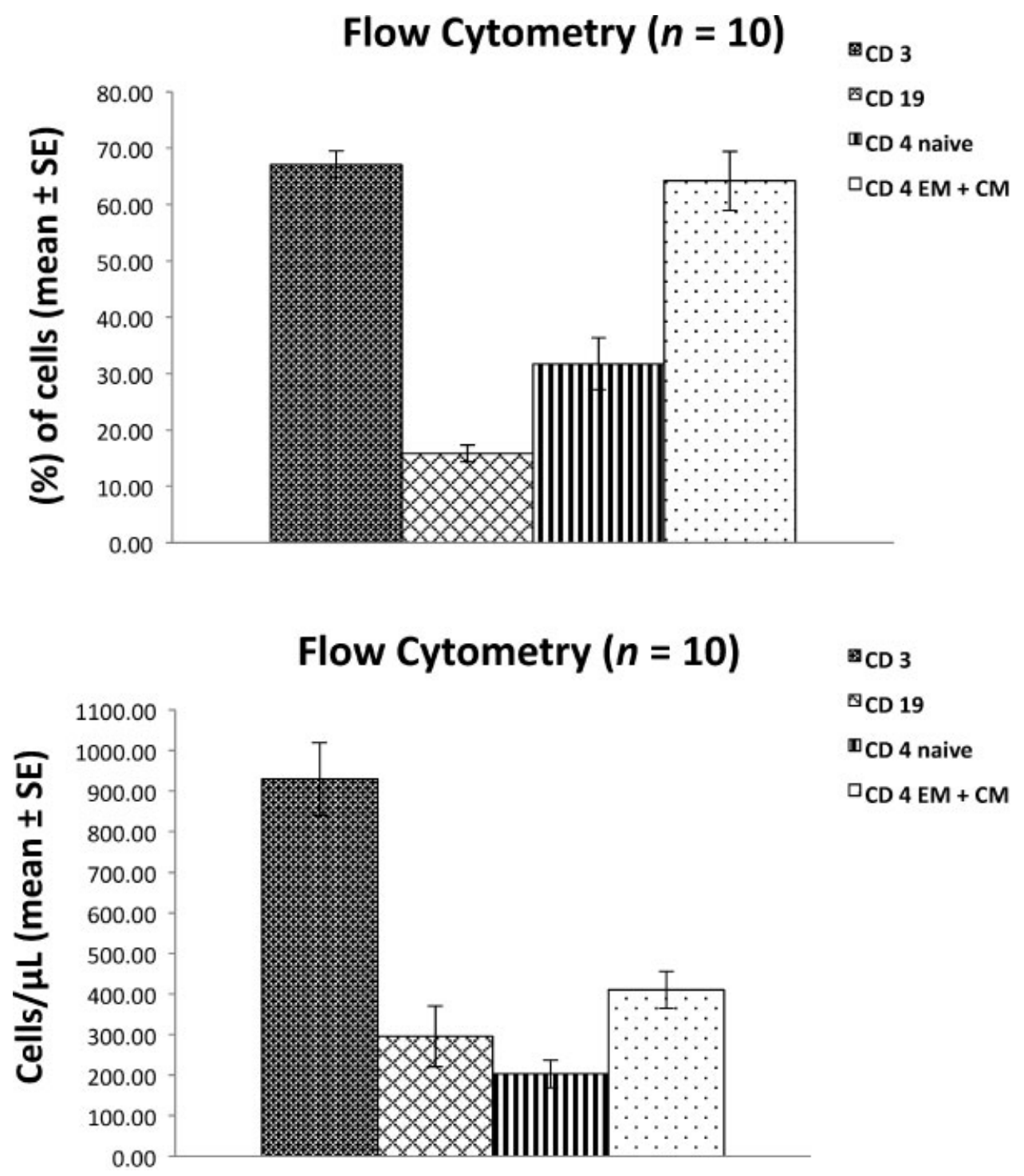

Fig. 3 Flow cytometry for B and T cell activity collected from patient serum at the time of revision surgery. Top graph demonstrates the percentage of the cells present. Bottom graph demonstrates the quantity of cells present per microliter.

\section{Flow Cytometry $(n=14)$}

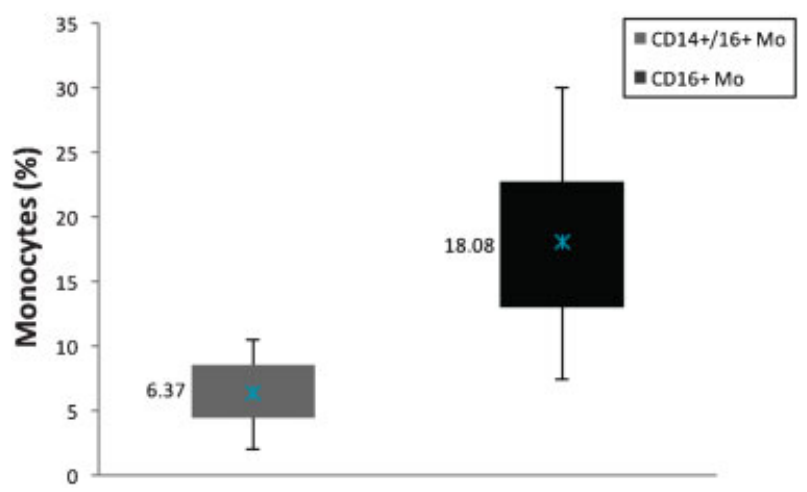

Fig. 4 Peripheral monocytes $\mathrm{CD} 14^{+} / \mathrm{CD} 16^{+}$and total $\mathrm{CD} 16^{+}$population are presented for $14 / 21$ patients. implant design, and/or cement preparation. Their revisions occurred at an average follow-up of 17 months (range: 9-31 months). While the most likely explanation for "early" mechanical loosening is surgical and/or cementing technique, our cohort had revisions at an average of $6.8 \pm 3.2$ years that suggests that late mechanical loosening can occur at any time point. Our study aimed to investigate whether this unique failure pattern and patient cohort had an inflammatory response that may have exacerbated or contributed to the failure. In that way, this is the first study of its kind. The biologic analysis indicated that the failure pattern was not that which is typically seen in inflammatory-induced loosening where patients have elevated levels of IL-1, TNF- $\alpha$, and IL-6. The levels of inflammatory markers IL- 1 and TNF- $\alpha$ were low in our patient cohort. The serum IL-6 levels in were $130.96 \pm 72.75$ (SE) $\mathrm{pg} / \mathrm{mL}$ (range: $0-1,182 \mathrm{pg} / \mathrm{mL}$ ) with a median value of 
$19.09 \mathrm{pg} / \mathrm{mL}$. Gandhi et al reported similar serum IL-6 values in patients with knee pain following TKA. ${ }^{30}$ IL-6 is promoted by osteoclasts and our wide range of values herein could represent a healing response due to the tibial bone collapse/fractures observed on the medial tibia. ESR and WBC levels were normal for all patients and CRP was only slightly elevated in a few patients. However, joint fluid analysis, fluid culture, and intraoperative assessment all indicated aseptic failures. Peripheral blood monocytes obtained from 14/21 patients showed that total CD16 ${ }^{+}$monocytes were $18.08 \pm 6.79 \% \mathrm{SD}$. This is comparable to findings reported by Wu et al which determined mean percentages of inflammatory total CD16 ${ }^{+}$ monocyte values in four groups: mechanical loosening patients $(14.0 \pm 6.3 \%)$, stable implant $(15.8 \pm 5.7 \%)$, osteoarthritis patients ( $16.7 \pm 6.3 \%)$, and AL patients $(22.4 \pm 10.6 \%)$ $(p<0.05) \cdot{ }^{31}$ These data would similarly point to a mechanical failure pattern.

The study is not without limitations. While the data were collected prospectively as part of a larger revision database collection protocol, the current study data were retrieved to evaluate what appeared to be a small, but unique, cohort of patients. As a result, not all of the patients had complete flow cytometric data. Also, the alignment presented in our study was obtained using short leg films. Although, short leg films can address tibial component alignment in comparison to tibial shaft, this should not serve as a surrogate to overall limb alignment. Finally, we were not able to present a control group with our results because the inflammatory biomarkers were only collected at the time of revision surgery. We, therefore, did not have inflammatory biomarker profiles on patients with functioning Stryker Scorpio knees, but the variation of the biomarker profile when compared with knees that failed as a result of wear debris was evident. Of note, this was a very small group of failures from at least 2,000 Scorpio implants performed by the senior author during the collection period. Unfortunately, we are unable to present an accurate revision rate for the implant system as we did not have a state-wide registry at the time. The anecdotal revision rate was as noted, low, with these cases derived from a primary series in excess of 2,000 . The data on the revisions represented herein were obtained from our laboratories revision data bank that contained 157 cases that were contributed by the senior author in the same time period.

Our purpose was to characterize the radiographic failure pattern, investigate the biologic response, and determine if the failure mechanism of these implants was of a biological or a mechanical nature. The failure pattern was consistently tibia vara and the loosening mechanism seemed to be mechanical based on the variation in the inflammatory biomarker profile. The biologic response was rather benign and did not resemble that typically seen with wear debrisinduced inflammation. Continued exploration into implant failure mechanisms and particularly into the biologic response associated with failure is ongoing. The data herein offer some insight into a unique cohort of patients with what appeared to be an unusual mechanical failure pattern. The lessons may be applied to other implants moving forward.

\section{Conclusion}

A small and unique patient cohort was identified with tibial implant failure. We speculated based on the findings presented in this article that the failure mechanism was mechanical, as there was lack of the characteristic biological response seen in wear debris-induced AL. We theorize that tibial component failure may be related to surgical or cementing technique, stresses due to the knees favorable rotational nonconstraints, or patient characteristics and behavior.

Conflict of Interest

None declared.

\section{References}

1 Callahan CM, Drake BG, Heck DA, Dittus RS. Patient outcomes following tricompartmental total knee replacement. A metaanalysis. JAMA 1994;271(17):1349-1357

2 Dusad A, Pedro S, Mikuls TR, et al. Impact of total knee arthroplasty as assessed using patient-reported pain and health-related quality of life indices: rheumatoid arthritis versus osteoarthritis. Arthritis Rheumatol 2015;67(09):2503-2511

3 Seo J-G, Moon YW, Cho BC, et al. Is total knee arthroplasty a viable treatment option in octogenarians with advanced osteoarthritis? Knee Surg Relat Res 2015;27(04):221-227

4 Stone OD, et al. Severe arthritis predicts greater improvements in function following total knee arthroplasty. Knee Surg Sports Traumatol Arthrosc 2017;25(08):2573-2579

5 Kurtz S, Ong K, Lau E, Mowat F, Halpern M. Projections of primary and revision hip and knee arthroplasty in the United States from 2005 to 2030. J Bone Joint Surg Am 2007;89(04):780-785

6 Abbas D, Gunn RS. Medium-term results of the Scorpio total knee replacement. Knee 2006;13(04):307-311

7 Borrione F, Bonnevialle P, Mabit C, et al. Scorpio single radius total knee arthroplasty. A minimal five-year follow-up multicentric study. Int Orthop 2011;35(12):1777-1782

8 Bozic KJ, Kinder J, Meneghini RM, Zurakowski D, Rosenberg AG, Galante JO. Implant survivorship and complication rates after total knee arthroplasty with a third-generation cemented system: 5 to 8 years followup. Clin Orthop Relat Res 2005;(435): 277

9 Colwell CW Jr, Gelber JD, Pulido PA, Casey KM. Early range of motion of the scorpio non-restrictive geometry cruciate-retaining total knee system. J Arthroplasty 2011;26(05):751-755

10 Kolisek FR, Barnes CL. Scorpio posterior-stabilized knee system: 5-year clinical and functional results. J Arthroplasty 2006;21(08): $1187-1192$

11 Mahoney OM, Kinsey TL. 5- to 9-year survivorship of singleradius, posterior-stabilized TKA. Clin Orthop Relat Res 2008;466 (02):436-442

12 Pandit H, van Duren BH, Price M, et al. Constraints in posteriorstabilised TKA kinematics: a comparison of two generations of an implant. Knee Surg Sports Traumatol Arthrosc 2013;21(12): 2800-2809

13 Martin A, Quah C, Syme G, Lammin K, Segaren N, Pickering S. Long term survivorship following Scorpio total knee replacement. Knee 2015;22(03):192-196

14 Silva M, Kabbash CA, Tiberi JV III, et al. Surface damage on open box posterior-stabilized polyethylene tibial inserts. Clin Orthop Relat Res 2003;(416):135-144

15 Pajarinen J, Jamsen E, Konttinen YT, Goodman SB. Innate immune reactions in septic and aseptic osteolysis around hip implants. J Long Term Eff Med Implants 2014;24(04):283-296

16 González O, Smith RL, Goodman SB. Effect of size, concentration, surface area, and volume of polymethylmethacrylate particles on 
human macrophages in vitro. J Biomed Mater Res 1996;30(04): 463-473

17 Shanbhag AS, Jacobs JJ, Glant TT, Gilbert JL, Black J, Galante JO. Composition and morphology of wear debris in failed uncemented total hip replacement. J Bone Joint Surg Br 1994;76(01):60-67

18 Abbas S, Clohisy JC, Abu-Amer Y. Mitogen-activated protein (MAP) kinases mediate PMMA-induction of osteoclasts. J Orthop Res 2003;21(06):1041-1048

19 Gallo J, Kamínek P, Tichá V, Riháková P, Ditmar R. Particle disease. A comprehensive theory of periprosthetic osteolysis: a review. Biomed Pap Med Fac Univ Palacky Olomouc Czech Repub 2002; 146(02):21-28

20 Passlick B, Flieger D, Ziegler-Heitbrock HW. Identification and characterization of a novel monocyte subpopulation in human peripheral blood. Blood 1989;74(07):2527-2534

21 Randolph GJ, Sanchez-Schmitz G, Liebman RM, Schäkel K. The CD16(+) (FcgammaRIII(+)) subset of human monocytes preferentially becomes migratory dendritic cells in a model tissue setting. J Exp Med 2002;196(04):517-527

22 Ren W, Jackson N, Song W, Shi T, Flynn JC, Markel DC. Implant wear induced inflammation is mitigated in CX3CR1(-/-) mice. J Orthop Res 2014;32(08):1037-1043

23 Childs LM, Goater JJ, O'Keefe RJ, Schwarz EM. Effect of anti-tumor necrosis factor- $\alpha$ gene therapy on wear debris-induced osteolysis. J Bone Joint Surg Am 2001;83-A(12):1789-1797

24 Ren W, Li XH, Chen BD, Wooley PH. Erythromycin inhibits wear debris-induced osteoclastogenesis by modulation of murine macrophage NF-kappaB activity. J Orthop Res 2004;22(01): 21-29

25 Ren W, Wu B, Peng X, et al. Erythromycin inhibits wear debrisinduced inflammatory osteolysis in a murine model. J Orthop Res 2006;24(02):280-290

26 Vanlommel J, Luyckx JP, Labey L, Innocenti B, De Corte R, Bellemans J. Cementing the tibial component in total knee arthroplasty: which technique is the best? J Arthroplasty $2011 ; 26(03)$ : 492-496

27 Valiathan R, Deeb K, Diamante M, Ashman M, Sachdeva N, Asthana D. Reference ranges of lymphocyte subsets in healthy adults and adolescents with special mention of $\mathrm{T}$ cell maturation subsets in adults of South Florida. Immunobiology 2014;219(07): 487-496

28 Arsoy D, Pagnano MW, Lewallen DG, Hanssen AD, Sierra RJ Aseptic tibial debonding as a cause of early failure in a modern total knee arthroplasty design. Clin Orthop Relat Res 2013;471 (01):94-101

29 Foran JR, Whited BW, Sporer SM. Early aseptic loosening with a precoated low-profile tibial component: a case series. J Arthroplasty 2011;26(08):1445-1450

30 Gandhi R, Santone D, Takahashi M, Dessouki O, Mahomed NN. Inflammatory predictors of ongoing pain 2 years following knee replacement surgery. Knee 2013;20(05):316-318

31 Wu W, Zhang X, Zhang C, Tang T, Ren W, Dai K. Expansion of $\mathrm{CD} 14+\mathrm{CD} 16+$ peripheral monocytes among patients with aseptic loosening. Inflamm Res 2009;58(09):561-570 